\title{
Secondary mitral regurgitation repair techniques and outcomes: Subannular repair techniques in secondary mitral regurgitation type $\mathrm{IIIlb}$
}

Jonas Pausch, MD, ${ }^{\mathrm{a}}$ Evaldas Girdauskas, MD, ${ }^{\mathrm{b}}$ Lenard Conradi, $\mathrm{MD},{ }^{\mathrm{a}}$ and Hermann Reichenspurner, $\mathrm{MD}, \mathrm{PhD}{ }^{\mathrm{a}}$

Feature Editor's Note-Despite decades of experimental and clinical work, secondary mitral regurgitation is still a challenge for cardiac surgeons. The excellent outcomes achieved in the treatment of organic mitral disease are light years away for secondary mitral regurgitation. Treating secondary mitral disease is too often a humbling and frustrating experience for all the parties involved (most importantly the patients). We now know that techniques that were designed to treat valvular problems do not work well when the mechanism of the mitral regurgitation is within the left ventricle, but it is still unclear if mitral valve repair may or may not have a role in this setting. The review by Pausch and colleagues is a nice summary of the current technical options with an eye to the future and will be of great interest to all those interested in treating this challenging and humbling disease.

\section{Mario Gaudino, MD, PhD, MSCE}

Secondary mitral regurgitation (SMR) due to left ventricular (LV) remodeling is a common sequel of chronic heart failure and associated with dismal outcome. In addition to optimal medical therapy (OMT), both surgical mitral valve repair and replacement are therapeutic options for selected patients. Isolated mitral annuloplasty is associated with increased rates of recurrent SMR during long-term follow-up and has not shown any prognostic benefit in comparison with chordal-sparing mitral valve replacement (MVR). ${ }^{1}$ Nevertheless, to avoid prosthesis-related complications (eg, increased perioperative mortality, endocarditis, thromboembolism, etc), additional subannular repair techniques, specifically addressing mitral leaflet tethering, have been developed to improve long-term mitral valve competence. We herein review the current subannular treatment options, with a special emphasis on the technique of

From the ${ }^{a}$ Department of Cardiovascular Surgery University Heart \& Vascular Center Hamburg, Hamburg; and ${ }^{\mathrm{b}}$ Department of Cardiothoracic Surgery, University Hospital Augsburg, Augsburg, Germany.

Received for publication July 29, 2021; accepted for publication Sept 2, 2021; available ahead of print Sept 16, 2021.

Address for reprints: Hermann Reichenspurner, $\mathrm{MD}, \mathrm{PhD}$, University Heart \& Vascular Center Hamburg, Martinistraße 52, D-20246 Hamburg, Germany (E-mail: reichenspurner@uke.de).

JTCVS Techniques 2021;10:92-7

2666-2507

Copyright (C 2021 The Author(s). Published by Elsevier Inc. on behalf of The American Association for Thoracic Surgery. This is an open access article under the CC BY-NC-ND license (http://creativecommons.org/licenses/by-nc-nd/4.0/).

https://doi.org/10.1016/j.xjtc.2021.09.019

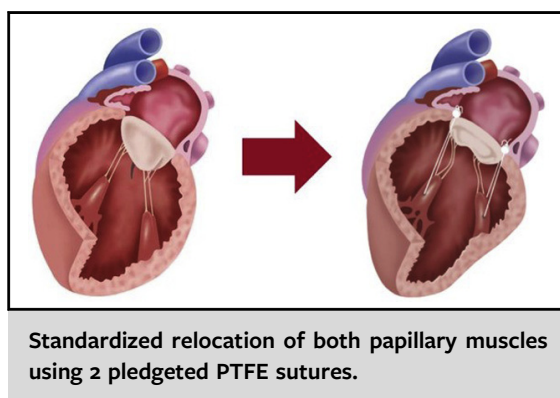

CENTRAL MESSAGE

Standardized relocation of both papillary muscles in addition to ring annuloplasty to correct SMR type IIIb specifically addresses mitral leaflet tethering, potentially improving long-term durability.

See Commentaries on pages 98 and 100.

standardized papillary muscle relocation (Figure 1) and its potential prognostic benefits in the treatment of SMR type IIIb.

\section{PATHOGENESIS AND PROGNOSIS OF SMR}

According to Alain Carpentier's classification of mitral regurgitation, which is based on mitral leaflet motion, SMR includes 2 different pathophysiologic entities. ${ }^{2}$ SMR with normal leaflet motion (type I) is mainly caused by isolated mitral annular dilatation due to atrial remodeling and is therefore also referred to as "atrial SMR." ${ }^{3}$ Most notably in patients with long-lasting permanent atrial fibrillation, excessive atrial dilatation and consecutive mitral annular dilatation lead to a central coaptation defect of the mitral leaflets, mostly without $\mathrm{LV}$ dysfunction. ${ }^{4}$

On the contrary, SMR with restricted leaflet motion during systole (type IIIb) is the result of progressive LV remodeling caused by ischemic or dilated cardiomyopathy (DCM) and is therefore also referred to as "ventricular SMR." Accompanying chronic heart failure, progressive global LV dilatation as well as regional cardiac remodeling promotes LV geometric distortion. Thereby, the apicolateral 

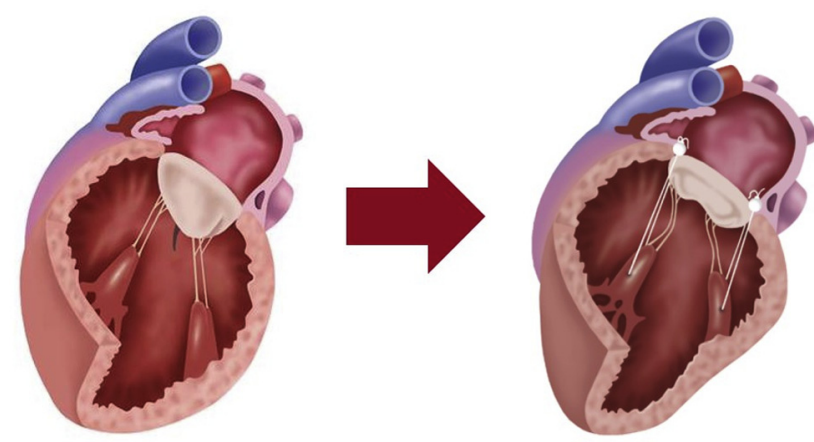

FIGURE 1. Standardized relocation of both papillary muscles. Additional standardized relocation of both papillary muscles using 2 pledgeted polytetrafluoroethylene sutures to resolve mitral leaflet tethering in the treatment of secondary mitral regurgitation type IIIb.

displacement of both papillary muscles leads to mitral leaflet tethering (Figure 2) and restrictive leaflet motion during systole. ${ }^{5}$ Consecutively increased LV volume overload further promotes diastolic wall stress, LV dilatation, and worsening of heart failure. ${ }^{6}$ Furthermore, SMR type IIIb, which is prevalent in about one-third of patients with advanced heart failure, ${ }^{7}$ is associated with excessive mortality, independent of the degree of LV dysfunction. ${ }^{8}$

\section{SURGICAL THERAPEUTIC OPTIONS FOR SMR TYPE IIIB}

Regarding their differing pathophysiologic background and prognosis, ${ }^{9}$ surgical therapeutic options for SMR type I and type IIIb should be tailored accordingly. In contrast to pure annular dilatation (SMR type I), the pathogenetic key factors of SMR type IIIb, namely LV distortion and mitral leaflet tethering (Figure 2), are not addressed by isolated mitral annuloplasty. Consequently, isolated mitral annuloplasty, as a treatment option for SMR type IIIb, is associated with significant rates of recurrent SMR (ie, up to $60 \%$ ) after 1 to 2 years postoperatively ${ }^{1}$ and does not
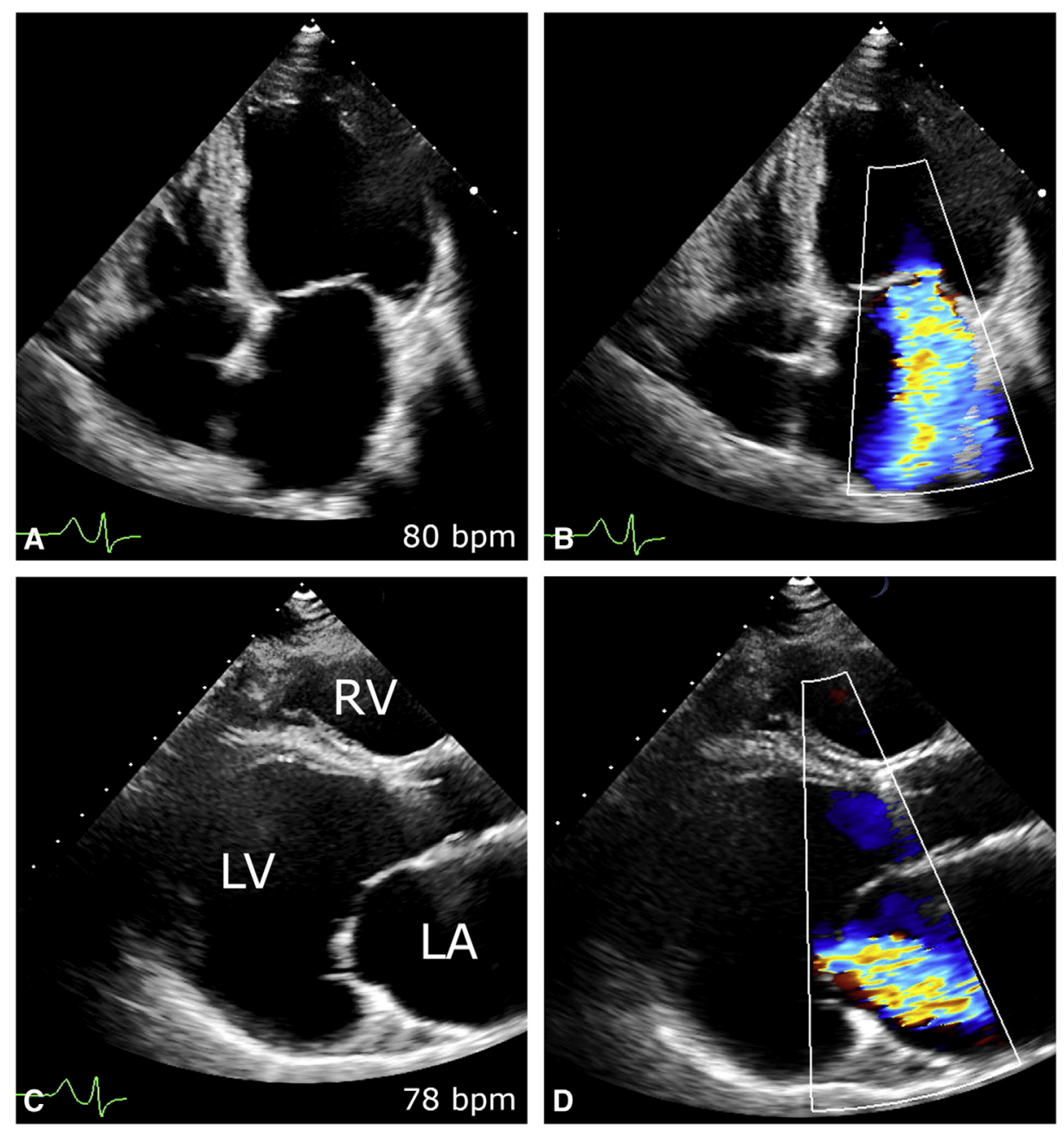

FIGURE 2. Dilated cardiomyopathy with associated SMR type IIIb due to mitral leaflet tethering. Preoperative transthoracic echocardiogram. Severe SMR type IIIb due to advanced LV distortion and mitral leaflet tethering in apical 4-chamber (A, B) and parasternal long-axis view (C, D). $R V$, Right ventricle; $L V$, left ventricle; $L A$, left atrium. 
provide any additional benefits in comparison with OMT. ${ }^{10}$ Furthermore, although chordal-sparing MVR is associated with an increased perioperative mortality ${ }^{11}$ long-term survival after 2 years is similar in comparison to isolated annuloplasty. ${ }^{1}$ Nevertheless, patients without recurrent SMR after isolated annuloplasty showed significantly increased reverse cardiac remodeling in comparison with MVR. ${ }^{1}$ Therefore, in an effort, to improve the long-term durability after mitral valve repair and to avoid the potential drawbacks of MVR, additional subannular repair strategies have been established.

\section{SECONDARY "CHORDAL CUTTING"}

Due to the geometric displacement of both papillary muscles, the attached chordae mediate mitral leaflet tethering, thereby disrupting mitral leaflet coaptation. To improve mitral leaflet mobility and resolve leaflet tethering, the division of tethered secondary chordae was designed. ${ }^{12}$ Borger and colleagues ${ }^{13}$ reported their initial results, comparing additional division of secondary chordae with isolated annuloplasty in the treatment of ischemic SMR type IIIb. According to the preoperative echocardiographic determination of the ischemic papillary muscle, the arising tethered secondary chordae were identified intraoperatively and divided in addition to downsizing annuloplasty. Although periprocedural complication rates were similar and a significant reduction of leaflet tethering was demonstrated early postoperatively within the "chordal cutting" group, no additional benefits regarding 2-year survival or event-free-survival were achieved. Nevertheless, Calafiore and coworkers ${ }^{14}$ demonstrated in a propensitymatched study that additional chordal cutting was associated with reduced rates of recurrent SMR during a follow-up of 33 months in comparison with isolated annuloplasty. Of note, patients with advanced preoperative leaflet tethering (tenting height $>10 \mathrm{~mm}$ ) were excluded and neither long-term survival nor other clinical end points were analyzed. On the contrary, according to Murashita and coworkers, ${ }^{15}$ long-term recurrence rates of severe SMR were comparably high despite additional chordal cutting in comparison with isolated annuloplasty, especially in patients with excessive preoperative leaflet tethering. Due to the progression of $\mathrm{LV}$ distortion and the existence of further sets of chordae, secondary chordal cutting might only temporarily reduce mitral leaflet tethering and not prevent from recurrent SMR in the long run. Furthermore, disturbance of the valvular-ventricular continuity potentially resulting from chordal cutting might even decrease LV function and prevent $\mathrm{LV}$ reverse cardiac remodeling. ${ }^{16}$

Therefore, despite its simplicity and feasibility, convincing evidence of a beneficial long-term effect of additional chordal cutting to treat of SMR type IIIb in comparison with isolated annuloplasty is still missing.

\section{MITRAL LEAFLET AUGMENTATION}

The loss of mitral leaflet mobility and coaptation surface due to mitral leaflet tethering promotes recurrent SMR after isolated annuloplasty. ${ }^{17}$ Therefore, additional leaflet patch augmentation was designed to restore leaflet mobility and increase coaptation surface. ${ }^{18}$ According to Kincaid and coworkers, ${ }^{19}$ the enlargement of the anterior mitral leaflet using an elliptically shaped $1 \times 3-\mathrm{cm}$ bovine pericardial patch in addition to mitral annuloplasty did not increase perioperative risk, whereas the rate of recurrent SMR after 2 years was low. Unfortunately, follow-up was only completed in $60 \%$ of the included patients, and the authors did not implement a control group of patients treated with isolated annuloplasty, nor did they give any information about defined inclusion criteria or echocardiographic tethering parameters.

de Varennes and coworkers ${ }^{20}$ implanted a bovine pericardial patch, extending from the medial half of P2 to the posterior commissure, in addition to ring annuloplasty. According to their initial results, no periprocedural complications related to leaflet augmentation occurred. Furthermore, promising low rates of recurrent MR after 1 year were achieved, despite absences of LV functional improvement or decrease of LV dimensions.

Circumferential augmentation of the posterior mitral leaflet using an autologous pericardial patch was associated with significantly reduced mitral leaflet tethering and an increased coaptation length immediately postoperatively in a single-center observational study with a limited number of patients by Ikeda and coworkers. ${ }^{21}$

Despite promising periprocedural results, with reduction of leaflet tethering, so far there are no studies available analyzing the long-term effects of additional mitral leaflet patch augmentation in comparison to isolated annuloplasty. Additionally, as there are no studies available addressing the durability of the inserted pericardial patch material, it remains debatable, whether mitral leaflet patch augmentation has any advantage in comparison with chordalsparing MVR. Although mitral leaflet patch augmentation is feasible, it remains a challenging operation, potentially increasing cardiac bypass and crossclamp times, and is therefore limited to individual cases treated in experienced heart valve centers.

\section{PAPILLARY MUSCLE APPROXIMATION (PMA)}

As LV distortion including papillary muscle displacement induces mitral leaflet tethering, several subannular repair strategies, focusing on the restoration of LV geometry and papillary muscle orientation, have been introduced. ${ }^{22}$

Besides apical displacement of both papillary muscles, the interpapillary muscle distance increases during progression of heart failure. ${ }^{23}$ Nappi and coworkers ${ }^{24}$ convincingly demonstrated within a randomized controlled trial that 
PMA using a Gore-Tex tube in addition to ring annuloplasty was associated with a reduction in the rate of recurrent SMR 5 years postoperatively in comparison with isolated annuloplasty. Although there was no intergroup difference regarding long-term survival, additional PMA was associated with an effective resolution of mitral leaflet tethering and increased LV reverse remodeling. ${ }^{24}$ A more invasive approach by Wakasa and coworkers ${ }^{25}$ demonstrated a survival benefit of complete PMA in ischemic SMR type IIIb using a pledgeted mattress suture via an LV incision in comparison with an incomplete PMA. Furthermore, Roshanali and coworkers ${ }^{26}$ showed a similar reduction of recurrent SMR after PMA in patients with DCM and consecutive SMR type IIIb. Of note, none of these procedures was applied in a minimally invasive mitral valve surgery setting.

\section{STANDARDIZED RELOCATION OF BOTH PAPILLARY MUSCLES}

The concept of an active surgical LV reverse remodeling procedure to resolve mitral leaflet tethering in ischemic SMR type IIIb was initially described by Liel-Cohen and colleagues. ${ }^{27}$ This experimental study not only emphasized causal relation of LV distortion and SMR type IIIb but illustrated the importance of papillary muscle tip-to-annulus distance in promoting mitral leaflet tethering. Therefore, apart from PMA, relocation of papillary muscles to reduce the tip-to-annulus distance in addition to ring annuloplasty was developed to specifically resolve leaflet tethering. ${ }^{28}$

In addition to ring annuloplasty (RING), Langer and coworkers $^{29}$ inserted a transventricular pledgeted suture (STRING), originating from the head of the posteromedial papillary muscle and then passed from the LV cavity through the aortomitral continuity underneath the commissure between the non- and left coronary aortic cusps and exteriorized. The exteriorized suture is tied beating heart with echocardiographic guidance, and mitral leaflet tethering is resolved using stepwise traction. ${ }^{29}$ Despite the resolution of mitral leaflet tethering and reduction of recurrent SMR, there was no long-term survival benefit in comparison with an historical control group of patients treated with isolated annuloplasty. Of note, full sternotomy and additional aortotomy is mandatory for the "RING and STRING technique."

Fattouch and coworkers ${ }^{30}$ found not only a resolution of mitral leaflet tethering and reduced rates of recurrent SMR after additional papillary relocation in comparison with isolated annuloplasty but achieved a reduced rate of adverse cardiac events during long-term follow-up. Of note, all patients underwent multivessel coronary artery bypass grafting $(\mathrm{CABG})$ via full-sternotomy, and none of the patients suffered from DCM.

To combine a reproducible subannular mitral valve repair procedure, aiming at durable results when treating SMR type IIIb, with the advantage of a minimally invasive mitral valve surgery setting via anterolateral minithoracotomy, we refined and simplified the concept of Kron and coworkers (Figure 1). ${ }^{28}$ Two pledgeted PTFE-sutures are placed through the trunks of both papillary muscles (Figure 3, A) and subsequently passed through the posterior mitral valve annulus (Figure 3, B). After ring annuloplasty is accomplished, both polytetrafluoroethylene sutures are placed through the posterior aspect of the annuloplasty ring (Figure 3,C). After the LV is maximally filled with cold saline, reduction of papillary muscle tip-to-annulus distance is achieved via stepwise traction on both polytetrafluoroethylene sutures, thereby mitral leaflet tethering completely disappears and both sutures are tightly knotted on the annuloplasty ring, while keeping traction (Figure $3, D){ }^{31}$ Besides a significant reduction of echocardiographic parameters of mitral leaflet tethering (tenting height $[\mathrm{mm}]$ : $12.4 \pm 2.3$ [preoperative] vs $5.3 \pm 1.3$ [1-year follow-up] $[P<.001]$; tenting area $\left[\mathrm{mm}^{2}\right]: 318 \pm 81.8$ [preoperative] vs $83.8 \pm 21.4$ [1-year follow-up] $[P<.001]$ ), additional papillary muscle relocation was associated with decreased rates of recurrent SMR as well as major adverse cardiac events 1 year postoperatively in comparison with isolated annuloplasty. ${ }^{32}$ Furthermore, we were able to demonstrate feasibility of standardized papillary muscle relocation in a minimally invasive mitral valve surgery setting, ${ }^{33}$ which is of particular interest for patients with ischemic dilated cardiomyopathy without the need of simultaneous CABG, or patients with DCM and associated SMR type IIIb. ${ }^{34}$ Interestingly, even without coronary revascularization, durable resolution of SMR type IIIb via papillary muscle relocation might promote LV reverse remodeling and therefore improve the long-term prognosis of selected heart failure patients. ${ }^{35}$

\section{TRANSCATHETER EDGE-TO-EDGE REPAIR FOR SMR}

Contradictory data regarding the prognostic effect of transcatheter edge-to-edge repair (TEER) in patients with heart failure has been published. ${ }^{36,37}$ Whereas the results of the COAPT (Cardiovascular Outcomes Assessment of the MitraClip Percutaneous Therapy for Heart Failure Patients with Functional Mitral Regurgitation) trial for the first time demonstrated that durable repair of SMR via TEER improves survival, promotes LV reverse remodeling, and reduces heart failure symptoms, results of the MITRA-FR (Percutaneous Repair with the MitraClip Device for Severe Functional/Secondary Mitral Regurgitation) trial showed no difference in comparison with OMT. In-depth comparison of both studies revealed that patients enrolled in the MITRA-FR trial had more advanced heart failure, more severe LV dilatation, and less SMR. Furthermore, the rate of recurrent SMR 1 year after TEER was greater within the MITRA-FR trial, which appears to be crucial for longterm prognosis. ${ }^{38}$ Unfortunately, no information about the 

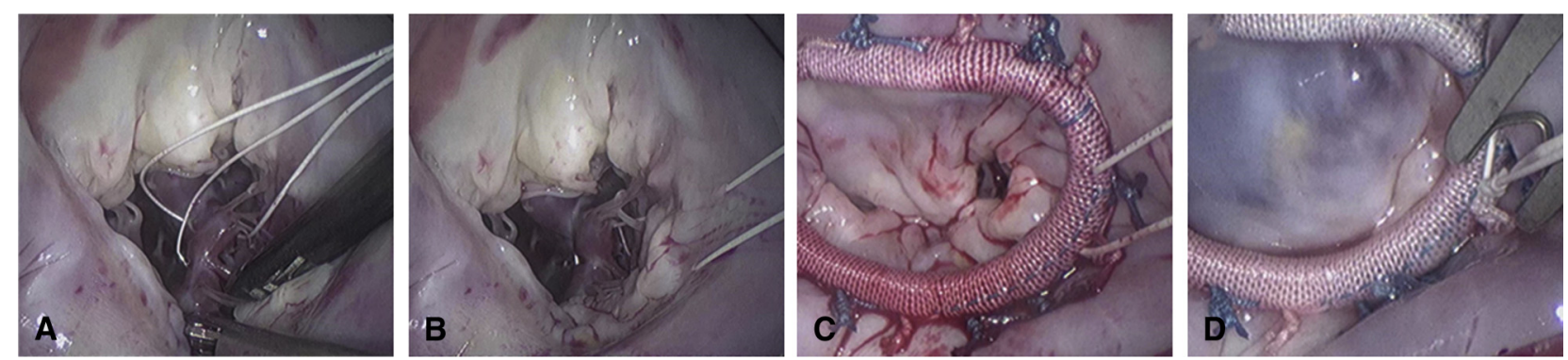

FIGURE 3. Standardized relocation of both papillary muscles. Exemplary intraoperative images of standardized relocation of the posteromedial papillary muscles in addition to mitral ring annuloplasty using a pledgeted 3-0 polytetrafluorethylene sutures.

predominant mechanism of SMR (type I: annular dilatation vs type IIIb: ventricular distortion) was given for either study. Whether advanced mitral leaflet tethering (eg, a tenting height $>10 \mathrm{~mm}$ ) resembling the origin of SMR type IIIb is durably restored using TEER needs to be addressed in future studies.

\section{CURRENT GUIDELINES ON THE TREATMENT OF SMR TYPE IIIB}

The management of patients with heart failure with SMR type IIIb is complex and needs to be optimized in a multidisciplinary heart team. Besides OMT, cardiac resynchronization therapy may promote reverse cardiac remodeling and therefore reduce SMR. ${ }^{39}$ Despite contradictory data on the prognostic benefits of TEER (COAPT vs MITRA-FR), this technique was upgraded within current North American guidelines. According to the 2020 American College of Cardiology/American Heart Association guidelines for the management of patients with valvular heart disease, ${ }^{40}$ in symptomatic patients despite OMT, with severe SMR, who do not require coronary revascularization and exhibit appropriate anatomical features (LV ejection fraction between $20 \%$ and $50 \%$; LV end-systolic dimension $<70 \mathrm{~mm}$; systolic pulmonary artery pressure $<70 \mathrm{~mm} \mathrm{Hg}$ ), TEER should be considered (Class IIa Level B), whereas mitral valve surgery may be considered (Class IIb Level B). ${ }^{40}$ Furthermore, only in selected patients with severe SMR who are symptomatic despite OMT and require coronary revascularization, simultaneous mitral valve surgery during CABG should be considered (Class IIa Level B). Due to increased rates of recurrent MR and missing evidence of a prognostic benefit after isolated annuloplasty, ${ }^{1}$ chordal-sparing mitral valve replacement may be considered instead of isolated annuloplasty (Class IIb Level B).

Of note, additional subannular repair techniques (eg, papillary muscle relocation), which are potentially more effective and durable, are so far not integrated within current guidelines. Furthermore, solid data comparing mitral valve surgery with TEER for treatment of SMR are still missing.

\section{CONCLUSIONS}

SMR includes 2 different pathophysiologic entities (type I vs type IIIb), and therapeutic options should be tailored accordingly. Despite OMT, SMR type IIIb has an adverse prognostic impact during the progression of chronic heart failure and needs to be addressed in selected patients. Isolated ring annuloplasty is associated with increased rates of recurrent SMR, has failed to show any additional benefits in comparison with OMT or MVR and should be avoided in the treatment of SMR type IIIb. Additional subannular repair procedures, specifically addressing LV geometry and papillary muscle orientation, may improve long-term durability and promote reverse cardiac remodeling after mitral valve repair. Standardized relocation of both papillary muscles (Figure 1) in addition to ring annuloplasty is feasible in a minimally invasive mitral valve surgery setting and may improve patient outcome in comparison with isolated annuloplasty. Although further long-term evaluation is needed, it appears to be a promising therapeutic option for patients with heart failure with associated SMR type IIIb.

In addition to clinical parameters, a detailed echocardiographic evaluation of the predominant SMR subtype (atrial vs ventricular SMR), the extent of mitral leaflet tethering, and LV distortion is mandatory to facilitate heart team decisions and determine the appropriate therapeutic strategy (eg, transcatheter edge-to-edge repair vs subannular mitral valve repair vs chordal-sparing MVR).

\section{Conflict of Interest Statement}

The authors reported no conflicts of interest.

The Journal policy requires editors and reviewers to disclose conflicts of interest and to decline handling or reviewing manuscripts for which they may have a conflict of interest. The editors and reviewers of this article have no conflicts of interest.

\section{References}

1. Goldstein D, Moskowitz AJ, Gelijns AC, Ailawadi G, Parides MK, Perrault LP et al. Two-year outcomes of surgical treatment of severe ischemic mitral regurgitation. N Engl J Med. 2016;374:344-53.

2. Carpentier A. Cardiac valve surgery - the "French correction". J Thorac Cardiovasc Surg. 1983;86:323-37. 
3. Deferm S, Bertrand PB, Verbrugge FH, Verhaert D, Rega F, Thomas JD, et al. Atrial functional mitral regurgitation: JACC review topic of the week. J Am Coll Cardiol. 2019;73:2465-76.

4. Kagiyama N, Mondillo S, Yoshida K, Mandoli GE, Cameli M. Subtypes of atrial functional mitral regurgitation: imaging insights into their mechanisms and therapeutic implications. JACC Cardiovasc Imaging. 2020;13:820-35.

5. Asgar AW, Mack MJ, Stone GW. Secondary mitral regurgitation in heart failure: pathophysiology, prognosis, and therapeutic considerations. J Am Coll Cardiol. 2015;65:1231-48

6. Nasser R, Van Assche L, Vorlat A, Vermeulen T, Van Craenenbroeck E, Conraads V, et al. Evolution of functional mitral regurgitation and prognosis in medically managed heart failure patients with reduced ejection fraction. JACC Heart Fail. 2017:5:652-9.

7. Varadarajan P, Sharma S, Heywood JT, Pai RG. High prevalence of clinically silent severe mitral regurgitation in patients with heart failure: role for echocardiography. J Am Soc Echocardiogr. 2006;19:1458-61.

8. Goliasch G, Bartko PE, Pavo N, Neuhold S, Wurm R, Mascherbauer J, et al. Refining the prognostic impact of functional mitral regurgitation in chronic heart failure. Eur Heart J. 2018;39:39-46.

9. Okamoto C, Okada A, Nishimura K, Moriuchi K, Amano M, Takahama H, et al. Prognostic comparison of atrial and ventricular functional mitral regurgitation. Open Heart. 2021;8:e001574.

10. Wu AH, Aaronson KD, Bolling SF, Pagani FD, Welch K, Koelling TM. Impact of mitral valve annuloplasty on mortality risk in patients with mitral regurgitation and left ventricular systolic dysfunction. J Am Coll Cardiol. 2005;45:381-7.

11. Vassileva CM, Boley T, Markwell S, Hazelrigg S. Meta-analysis of short-term and long-term survival following repair versus replacement for ischemic mitral regurgitation. Eur J Cardiothorac Surg. 2011;39:295-303.

12. Messas E, Guerrero JL, Handschumacher MD, Conrad C, Chow CM, Sullivan S, et al. Chordal cutting: a new therapeutic approach for ischemic mitral regurgitation. Circulation. 2001;104:1958-63.

13. Borger MA, Murphy PM, Alam A, Fazel S, Maganti M, Armstrong S, et al. Initial results of the chordal-cutting operation for ischemic mitral regurgitation. $J$ Thorac Cardiovasc Surg. 2007;133:1483-92.

14. Calafiore AM, Refaie R, Iaco AL, Asif M, Al Shurafa HS, Al-Amri H, et al. Chordal cutting in ischemic mitral regurgitation: a propensity-matched study. $J$ Thorac Cardiovasc Surg. 2014;148:41-6.

15. Murashita T, Okada Y, Kanemitsu H, Fukunaga N, Konishi Y, Nakamura K, et al. Midterm outcomes of chordal cutting in combination with downsized ring annuloplasty for ischemic mitral regurgitation. Ann Thorac Cardiovasc Surg. 2014; 20:1008-15.

16. Rodriguez F, Langer F, Harrington KB, Tibayan FA, Zasio MK, Cheng A, et al. Importance of mitral valve second-order chordae for left ventricular geometry, wall thickening mechanics, and global systolic function. Circulation. 2004; 110(11 suppl 1):II115-22.

17. Hung J, Papakostas L, Tahta SA, Hardy BG, Bollen BA, Duran CM, et al. Mechanism of recurrent ischemic mitral regurgitation after annuloplasty: continued LV remodeling as a moving target. Circulation. 2004;110(11 suppl 1):II85-90.

18. Langer F, Rodriguez F, Cheng A, Ortiz S, Nguyen TC, Zasio MK, et al. Posterior mitral leaflet extension: an adjunctive repair option for ischemic mitral regurgitation? J Thorac Cardiovasc Surg. 2006;131:868-77.

19. Kincaid EH, Riley RD, Hines MH, Hammon JW, Kon ND. Anterior leaflet augmentation for ischemic mitral regurgitation. Ann Thorac Surg. 2004;78: 564-8; discussion 8 .

20. de Varennes B, Chaturvedi R, Sidhu S, Cote AV, Shan WL, Goyer C, et al. Initial results of posterior leaflet extension for severe type IIIb ischemic mitral regurgitation. Circulation. 2009;119:2837-43.

21. Ikeda N, Yamaguchi H, Takagaki M, Mitsuyama S, Ebato M, Tanno K, et al. Extended posterior leaflet augmentation for ischemic mitral regurgitationaugmented posterior leaflet snuggling up to anterior leaflet. Circ J. 2019;83:567-75.

22. Harmel EK, Reichenspurner H, Girdauskas E. Subannular reconstruction in secondary mitral regurgitation: a meta-analysis. Heart. 2018;104:1783-90.

23. Kim K, Kaji S, An Y, Nishino T, Tani T, Kitai T, et al. Interpapillary muscle distance independently affects severity of functional mitral regurgitation in patients with systolic left ventricular dysfunction. J Thorac Cardiovasc Surg. 2014;148: 434-40.e1.
24. Nappi F, Lusini M, Spadaccio C, Nenna A, Covino E, Acar C, et al. Papillary muscle approximation versus restrictive annuloplasty alone for severe ischemic mitral regurgitation. J Am Coll Cardiol. 2016;67:2334-46.

25. Wakasa S, Kubota S, Shingu Y, Ooka T, Tachibana T, Matsui Y. The extent of papillary muscle approximation affects mortality and durability of mitral valve repair for ischemic mitral regurgitation. J Cardiothorac Surg. 2014;9:98.

26. Roshanali F, Vedadian A, Shoar S, Naderan M, Mandegar MH. Efficacy of papillary muscle approximation in preventing functional mitral regurgitation recurrence in high-risk patients with ischaemic cardiomyopathy and mitral regurgitation. Acta Cardiol. 2013;68:271-8.

27. Liel-Cohen N, Guerrero JL, Otsuji Y, Handschumacher MD, Rudski LG Hunziker PR, et al. Design of a new surgical approach for ventricular remodeling to relieve ischemic mitral regurgitation: insights from 3-dimensional echocardiography. Circulation. 2000;101:2756-63.

28. Kron IL, Green GR, Cope JT. Surgical relocation of the posterior papillary muscle in chronic ischemic mitral regurgitation. Ann Thorac Surg. 2002;74 $600-1$.

29. Langer F, Kunihara T, Hell K, Schramm R, Schmidt KI, Aicher D, et al RING+STRING: successful repair technique for ischemic mitral regurgitation with severe leaflet tethering. Circulation. 2009;120(11 suppl):S85-91.

30. Fattouch K, Lancellotti P, Castrovinci S, Murana G, Sampognaro R, Corrado E, et al. Papillary muscle relocation in conjunction with valve annuloplasty improve repair results in severe ischemic mitral regurgitation. J Thorac Cardiovasc Surg. 2012;143:1352-5.

31. Girdauskas E, Conradi L, Harmel EK, Reichenspurner H. Minimally invasive mitral valve annuloplasty with realignment of both papillary muscles for correction of Type IIIb functional mitral regurgitation. Innovations (Phila). 2017;12: 329-32.

32. Harmel E, Pausch J, Gross T, Petersen J, Sinning C, Kubitz J, et al. Standardized subannular repair improves outcomes in type IIIb functional mitral regurgitation. Ann Thorac Surg. 2019;108:1783-92.

33. Pausch J, Harmel E, Sinning C, Reichenspurner H, Girdauskas E. Standardized subannular repair for type IIIb functional mitral regurgitation in a minimally invasive mitral valve surgery setting. Eur J Cardiothorac Surg. 2019;56: 968-75.

34. Pausch J, Sequeira Gross T, Muller L, von Stumm M, Kloth B, Reichenspurner H, et al. Subannular repair for functional mitral regurgitation type IIIb in patients with ischaemic versus dilated cardiomyopathy. Eur J Cardiothorac Surg. 2021; 60:122-30.

35. Pausch J, Sequeira Gross T, Reichenspurner H, Girdauskas E. Left ventricular reverse remodeling after successful subannular mitral valve repair in end-stage heart failure: a case report. Eur Heart J Case Rep. 2020;4:1-5.

36. Stone GW, Lindenfeld J, Abraham WT, Kar S, Lim DS, Mishell JM, et al. Transcatheter mitral-valve repair in patients with heart failure. N Engl J Med. 2018; 379:2307-18.

37. Obadia JF, Messika-Zeitoun D, Leurent G, Iung B, Bonnet G, Piriou N, et al Percutaneous repair or medical treatment for secondary mitral regurgitation. $N$ Engl J Med. 2018;379:2297-306.

38. Reichart D, Kalbacher D, Rubsamen N, Tigges E, Thomas C, Schirmer J, et al. The impact of residual mitral regurgitation after MitraClip therapy in functional mitral regurgitation. Eur J Heart Fail. 2020;22:1840-8.

39. Ghio S, Freemantle N, Scelsi L, Serio A, Magrini G, Pasotti M, et al. Long-term left ventricular reverse remodelling with cardiac resynchronization therapy: results from the CARE-HF trial. Eur J Heart Fail. 2009; 11:480-8.

40. Otto CM, Nishimura RA, Bonow RO, Carabello BA, Erwin JP III, Gentile F, et al. 2020 ACC/AHA guideline for the management of patients with valvular heart disease: executive summary: a report of the American College of Cardiology/ American Heart Association joint committee on clinical practice guidelines. Circulation. 2021;143:e35-71.

Key Words: functional mitral regurgitation, secondary mitral regurgitation, mitral leaflet tethering, subvalvular mitral valve repair, papillary muscle relocation, chronic heart failure 